\title{
La critique de la déraison économique chez Michel Henry*
}

\author{
Jean LECLERCQ \\ Université catholique de Louvain
}

Argent gagné, emprunté, reçu, dépensé, épargné, nous n’avons pas/jamais d'adjectifs en trop pour décrire notre rapport à ce mot qui est, le plus souvent, l'objet de nos fantasmes, de nos plus terribles fétichisations - dont la plus folle est sans doute la spéculation -, mais aussi l'objet de nos plus grands désarrois ou ... de nos plus grandes espérances. Comme le pense Paul Ricœur, « il est peu probable qu'aucun d'entre nous soit au clair quant à ses rapports avec l'argent. Nos rapports sont divers, contradictoires, enchevêtrés » ${ }^{1}$. Georg Simmel, lui-même, notait que «bien des gens sont enclins à se comporter avec beaucoup moins de conscience morale et de manière plus louche dans de pures affaires d'argent que lorsqu'il s'agit de faire quelque chose de douteux éthiquement dans d'autres relations $»^{2}$.

C'est donc sur ce terrain, diablement miné, entre concepts et affects, que nous allons déployer notre contribution, sachant que - très paradoxalement d'ailleurs - si le capitalisme fait l'objet d'une vaste littérature, il y en a moins sur l'argent, qui est souvent laissé à son statut empirico-objectif d'entité physique permettant d'établir un ordre de valeurs, à partir de considérations multiples (empiriques et théoriques), via le prix, attribué à des objets, des relations entre objets, et engageant donc des phénomènes multiples. Dans ce cas, l'argent est alors ici plutôt la monnaie, cet instrument quasi-universel, une norme de mesure et un moyen de commerce et d'échange. Mais cette focalisation sur les dimensions métallique, fiduciaire, scripturale - expressions de la portée mercantiliste de la notion - ne doit pas nous faire oublier que nous sommes, nous les Contemporains, entrés dans l'ère de la spéculation.

En effet, comme nous avons créé des bulles métaphysiques (Dieu, Absolu, etc.) qui nous ont menés dans des situations de fantasmes, de délires, d'ek-stases, en tout genre, et comme nous nous sommes également installés dans des apories sociétales dont nous ne parvenons d'ailleurs plus à nous extraire, tellement nos inventions nous piègent et nous déconnectent de la réalité de la matière affective réelle, nous avons

\footnotetext{
* Je dédie ce texte à mon ami Julien Uyttendaele dont j'ai la chance et l'honneur d'accompagner le parcours et l'action politiques.

${ }^{1}$ Paul Ricœur, « L’argent : d'un soupçon à l'autre », dans Esprit, numéro 1, 2010, p. 200.

${ }^{2}$ Georg Simmel, L'argent dans la culture moderne et autres essais sur l'économie de la vie, Laval, Presses de l’Université Laval (coll. « Pensée allemande et européenne »), 2006, p. 37.
} 
donc aussi créé des bulles financières dont les premières ont d'ailleurs déjà éclaté à nos yeux. Les déflagrations sont brutales, violentes, elles tuent parfois d'un coup ou alors elles asphyxient lentement, insidieusement.

Dans cette intervention qui est donc politiquement située et philosophiquement engagée, je voudrais procéder en trois temps : tout d'abord, je voudrais pointer quelques constats qui orientent obligatoirement vers la question du penser philosophique de l'argent et sa nécessité, puis je voudrais ensuite aborder la spécificité du traitement henryen de la question et, en usant de cette analyse, tirer une conclusion effective qui obligerait à penser politiquement l'argent, pour des raisons que j'expliciterai bien entendu.

\section{Constats : les affres de la réduction mercantile}

Nous sommes bel et bien entrés dans les affres des effets de la marchandisation totale et de la monétarisation générale, avec ces effets d'utilitarisme conséquents sur nos existences et nos modes de relation, mais aussi sur nos solidarités civiles et écologiques, nos fraternités, donc bien sûr la fiduciarité, la véritable car la plus existentielle, de nos liens sociaux. On nous parle de «l'économie », de la «raison économique " mais l'avez-vous déjà vue ? On nous dit qu'elle est une "science », mais alors reconnaissons qu'elle manque les objectifs de la définition du terme depuis la fondation de ses exigences chez les Grecs (universalité, causalité et nécessité) et avouons que ses catégories fondamentales et régulatrices sont problématiques.

C'est à cet égard qu'il convient d'abord de montrer que, sur la question de l'argent, il faut mesurer les enjeux du fait que, eu égard à sa fonction de médiateur de nos échanges, un des problèmes philosophiques (parce que c'est une question liée à l'usage des catégories) est l'inversion (déjà dénoncée par Simmel) de l'efficience et de la finalité, en sorte que les paradigmes opératoires de la raison calculatrice propre à l'économie sont remis en cause. C'est dire encore que la notion de quantité accède différemment à sa dimension abstraite et que l'argent devient ipso facto une finalité, dont rien n'est plus alors assuré qu'il soit articulé à son effectivité première, sachant bien sûr qu'il procède par une réduction à l'objet et qu'il porte, par tendance et potentiellement, la quantité au rang de valeur. En somme, on dira qu'il s'instaure une axiologie du/dans (le) quantitatif, au risque que, en raison du fait que la catégorie de quantité est liée à la satisfaction, le problème de l'aliénation devienne la question philosophique, en fait celle de la «Polis » puisque l'argent conquiert une valeur unique et influe le cours des relations sociales, en interrogeant bien sûr les mécanismes du don et de la dette.

C'est cela qu'il importe de penser philosophiquement : ce fait singulier que les besoins et les échanges deviennent l'essence de la manifestation du tout quantitatif 
et quantifiable que manifeste l'argent. La quantité, en son culte, devient alors la seule critériologie axiologique, voire une catégorie morale universelle.

Oc c’est en tant que lecteur assidu et éclairé de Marx que Michel Henry doit retenir notre attention sur ces différents points. Car Marx a voulu, très précisément, prendre à bras le corps ce problème de l'équation aliénation/abstraction, ce moment spécifique où la quantité accède au statut de valeur en soi et devient à la fois globalisante et totalisante, certes dans son herméneutique des échanges, mais aussi singulièrement dans les opérations de marchandisation.

A cet égard, il faut rappeler que Marx a décrit l'aliénation capitaliste en quatre temps $^{3}$ :

1. l'égoïsme de la propriété privée. Il implique que chacun doit posséder plus que les autres pour pouvoir s'affirmer ;

2. la création des objets nouveaux pour susciter de nouveaux désirs, de nouvelles jouissances. La dépendance intersubjective entre alors en excès ;

3. l'accroissement du nombre total d'objets ;

4. l'appauvrissement de l'homme comme homme, avec la perte des besoins vitaux et du « rapport réellement humain avec ses alter ego ».

C'est pour ces quatre raisons que l'argent est de plus en plus une nécessité, au gré d'un «cercle vicieux » dont la seule logique est celle de l'accroissement, celle d'avoir certes de plus en plus d'argent et corollairement celle de « susciter de nouveaux besoins ». Ces articulations étant rappelées, on peut désormais aborder le travail de Henry sur cette dimension.

\section{Le moment henryen : déconstruction des schèmes de l’aliénation}

Commençons par cette assertion extraite d'un entretien inédit de Henry mais récemment publié :

Et donc, on a construit un travail qui n'existe pas, qui est une entité idéale et qui est un objet économique. Car l'objet économique, ce n'est pas du tout le travail vivant. L’objet économique, c'est comme un objet géométrique. C’est un objet qui a été construit à partir de la réalité. Et aujourd’hui tout l’univers économique est construit ainsi. Parce que le travail abstrait ou économique, ou social, comme il le dit encore, est, lui, homogène à la valeur d'échange, homogène à l'argent... L’argent, la valeur d'échange, c'est du travail abstrait. Il y a donc tout un univers économique parfaitement homogène, et financier aussi, qui est constitué par des substituts de la vie réelle des gens. Et cet abîme est ouvert à jamais. ${ }^{4}$

\footnotetext{
${ }^{3}$ Je suis ici la contribution de Matthieu Dubost, « Argent et aliénation dans les Manuscrits de 1844 de K. Marx », dans Archives de Philosophie, numéro 3, 2008, p. 489-506.

${ }^{4}$ «Entretien inédit de juin 1996 avec Philippe Corcuff et Natalie Depraz », dans PV-V, p. 219-220.
} 
On voit en réalité combien Henry a cherché la concrétude du sujet corporel individué, voyant dans cette subjectivité sensible le «propre » de l'homme, et rejouant la distinction de l'empirique et du transcendantal, il a cherché dans l'affectivité de cette entité individuée sa marque quasi définitionnelle, le sceau anthropologique, son l'on veut. Nous ne reviendrons pas sur le geste de critique de la science et de l'empirisme biologique, avec les réductionnismes qu'ils impliquent, rendant l'homme impersonnel, cette « silhouette » errante dont Henry aime parler.

Pour lui, il est surtout une sensibilité, qui indivise et individualise tout à la fois, et qui est comprise par son «fond » dont il dit très vite qu'elle est la vie, cette entité absolue capable de générer par ses propres pouvoirs une identité concrète et affective. La modalité d'action se dit, chez Henry, via le champ sémantique de l'épreuve/expérience de soi, mais dont je tiens à dire d'emblée qu'elle ne se fait évidemment pas hors le monde et contre le monde (mais bien par contre «avant » le monde), pour annuler d'emblée toutes les lectures dénonçant un soi-disant hypertranscendantalisme chez Henry, au gré d'une herméneutique ou d'une lecture qui manquent de justesse à son égard.

Cette expérience affective, sans distance et sans écart, en raison du stade où elle est élucidée phénoménologiquement, est donc de nature pathétique et impressionnelle, en sorte qu'elle ressortit à une phénoménologie de l'intériorité radicale. Je ne reviendrai pas ici sur la question de la duplicité, du monisme ontologique, mais, pour le propos relatif au sujet de la contribution, j’insisterai juste sur ceci que ce corps compris dans sa subjectivité individuée et singularisée est précisément le locus de l'action. Si bien que j'ai pris l'habitude de ranger Henry parmi les philosophes de l'action.

Or ce lien entre subjectivité et action (et donc aussi le philosophème du pouvoir) est essentiel pour la question relevant de l'argent. En effet, c'est sur le lien ou l'axe praxis/argent que Henry joue sa posture, raison pour laquelle, par exemple, toute sa lecture de Marx sera une déconstruction de sa compréhension objectiviste, au prix d'une réinstauration d'une lecture subjectiviste. C'est donc la décision épistémique de penser l'action comme « vivante » (et donc radicalement réelle et historique par ce seul fait) et la volonté de penser les dimensions subjective, vivante et individuelle du travail qui donnent toute l'orientation au projet philosophique.

A cet égard, Henry peut très rapidement ranger l'économie du côté des sciences ou des savoirs de la réduction puisque celle-ci ne s'occupe pas du travail réel. En se basant sur une longue pratique de la lecture de Marx, il fait alors valoir que, comme il y a une duplicité phénoménologique de l'apparaître, il y a une duplicité du « travailler ». Un travail est alors compris en son objectivité : un travail qui produit une valeur d'échange réduite à l'argent (l'ordre de la représentation ou de l'abstraction) et un travail compris dans l'amplitude de sa subjectivité : une praxis du sujet individué vivant se réalisant par ses pouvoirs. A cet égard, le travail devient 
précisément l'actuation de la force subjective de l'individu, son « je peux » incarné et incorporé dans un sujet vivant.

Or cette définition spécifique reconduit l'équation travail/échange à un simplisme des théories de l'économie classique. En sorte que, pour Henry, «le problème de l'échange est un des grands problèmes métaphysiques de l'humanité ${ }^{5}$ et il ajoute même : «l'humanité préhistorique s'est posée deux problèmes métaphysiques fondamentaux : celui de la mesure du temps et celui de l'échange. ${ }^{6}$ En effet, dans les techniques de l'échange ( «du sel contre des peaux de bêtes »), le problème est bel et bien celui du «terme commun ». Or cette solution choisie oriente vers la temporalité, le « combien » de la fabrication qui devient conséquemment une valeur évaluative.

Mais, d'un autre côté, on doit cependant échanger des « produits du travail » et pas uniquement des choses ou des objets, permettant par là d'inclure une tonalité affective à la production (peine, fatigue, etc.), en sorte que chaque «travail » soit une expérience subjective. Mais alors au gré de quelle valence ? La souffrance, la peine, les forces, comment les évaluer ? Comment les faire rentrer dans un système de valeurs d'échanges?

Or tout le mouvement qui est analysé par Henry est celui où l'on passe du transfert du «travail réel, individuel, subjectif, vivant, invisible » au travail "abstrait, irréel », le travail de la construction économique (celui des « subjectivités muettes, indicibles et inconnaissables») devenant valeur d'échange. Du coup, explique Henry, « on a construit un travail qui n'existe pas, qui est une entité idéale et qui est un objet économique ${ }^{7}$. On quantifie le temps et on quantifie les affects de la production des travaux. More geometrico, si l'on veut.

De la sorte, chez Henry, c'est bien ce travail fictif qui est « homogène à l'argent », l'argent qui est «valeur d'échange » et «travail abstrait ». C'est donc un processus de substitution (mais aussi d'occultation de la réalité réelle, donc celle de la subjectivité) qui est à l'œuvre et crée les entités économiques et financières, séparées de la vie concrète et subjective, au point qu'elles sont capables de la démesure. L'enjeu, comme dit Henry, est donc d'œuvrer à la façon de " désolidarise(r) l'ensemble des quantifications et des qualifications objectives de la vie $"{ }^{8}$. C’est bien sûr ici l'utopie de la société sans mesure, celle de la «surabondance ».

Or, pour Henry, c'est évidemment ici que se repose la question de l'argent et de celle de savoir s'il peut être « valeur d'usage ». Il note ainsi que Marx a vu «que le travail vivant [...] était de moins en moins nécessaire à la production des biens de consommation, laquelle appartenait à une technique qui allait tendre à s'autonomiser

\footnotetext{
${ }^{5}$ Ibid., p. 217.

${ }^{6}$ Ibid.

${ }^{7}$ Ibid., p. 219.

${ }^{8}$ Ibid., p. 221.
} 
et qui était faite avec des processus objectifs empruntés à la nature » ${ }^{9}$. Et il ajoute : « À partir de ce moment-là, c'est toute l'économie du monde, depuis l'origine des temps, qui bascule. C'est-à-dire que la loi fondamentale qui reliait la valeur d'échange (c'est-à-dire l'argent) au travail vivant par la médiation du travail - et qu'on calculait par la médiation du travail abstrait, d'un travail fictif -, cette loi va disparaître. ${ }^{10}$

Le libre cours est donc donné à des « entités abstraites » : « auto-fonctionnement de l'économie », « auto-fonctionnement de la technique » et bien sûr l'absence de la « finalité humaine ». Par conséquent, pour Henry, il faut ré-enraciner ces entités dans la vie et lutter contre les effets de la délocalisation, de l'élimination des normes, de la destruction, et cela en un sens politique : «Et au fond, en gros, le politique est une façon d'appréhender l'activité humaine, qui est invisible, pathétique, singulière, vivante. ${ }^{11}$ Voilà pourquoi le jugement henryen est tranché : «Il y a totalitarisme quand le politique se pense comme essentiel au point d'oublier les individus. ${ }^{12}$

\section{Solutions, ouvertures et perspectives : revenir au Politique}

Il nous faut conclure. Ricœur considérait qu'il y avait trois strates dans notre imaginaire concernant l'argent : une strate morale, une strate économique et une strate politique. Il faut noter que le plan moral fait de l'argent un « moyen de paiement », un " signe abstrait », un " simple moyen d'échange ». Le plan politique, quant à lui, porte la question sur le thème des « sphères d'interaction humaine qui, à l'intérieur de l'enveloppe politique de la cité, échappent de droit à la mesure des biens sociaux premiers par la monnaie $"{ }^{13}$. C'est la grande question du non-marchand, de sa délimitation et de son effectivité, et donc la question de l'argent citoyen et de ses usages.

Or il faut retenir de la critique de l'aliénation l'urgence de mener une réflexion de fond sur la valeur des objets et des échanges, avec une tout autre axiologie que celle qui a inversé efficience et finalité. En sorte qu'il faudrait sans doute une " autre » pensée économique, voire une " économie de la vie »(Ôkonomie des Lebens), comme a voulu la penser Simmel.

Ceci dit, je tiens ultimement que

la morale tourne en effet en rond dès qu'elle se confronte à l'argent : elle en critique la fausse valeur, ses puissances d'illusion comme de corruption, l'amour qu'on lui porte comme la haine trop simpliste qu'on lui voue, mais pour en venir

\footnotetext{
${ }^{9}$ Ibid., p. 221.

${ }^{10}$ Ibid., p. 222.

${ }^{11}$ Ibid., p. 224.

${ }^{12}$ Ibid.

${ }^{13}$ Paul Ricœur, op. cit., p. 209.
} 
à le réhabiliter, tout en prétendant n’être pas dupe de ses effets les plus délétères, et donc finir par rechercher sans fin un juste milieu introuvable. ${ }^{14}$

Voilà pourquoi il n’est de réponse convaincante à la question de l'argent que celle du politique, en sorte que l'argent ne peut primer ou submerger sur/le politique qui doit rester le principe régulateur, le vecteur axiologique de cette sorte de philosophie première. Pourquoi ?

Je vois ici plusieurs champs d'action :

- la régulation des usages, via les circuits de production et de consommation, sachant qu'une monnaie reste toujours une production ;

- la nécessité de penser l'argent dans son rapport à la citoyenneté, pour lui garder cette « exigence d'invention collective », avec la préservation du lien social, en sorte de refuser les pratiques de réductions des individus à des pratiques d'exploitation, de domination, de fluctuation, avec les délocalisations et les déplacements afférents ;

- les dénonciations des logiques marchandes au profit de pratiques relevant d'un commerce équitable, ce lieu où la catégorie de qualité doit être réintroduite, via l'exigence de «produits de qualité », sur le plan de la matérialité, de l'utilité, du besoin, de la nature juste et sociale des échanges, des pratiques de redistribution, et ultimement d'un discours sur les fins supérieures qui ne doit pas refuser de regarder en face le véritable scandale qu'est la misère humaine ;

- la réflexion radicale et renouvelée sur les politiques fiscales, en termes de justice, d'équité, de solidarité, de redistribution et de cohésion ;

- la réflexion elle aussi très radicale sur la nécessité de libérer des domaines de la vie sociétale de liens d'effectivité avec l'argent, en sorte de «sanctuariser l'inévaluable », et singulièrement dans ces trois domaines que sont l'éducation, la culture et la santé, car elles sont les lignes de faîte de la Cité et elles sont les lieux de l'émancipation, du progrès social, de la fraternité civique et du caractère fondamentalement inévaluable (terme encore plus intensif que « nonmarchand ») de chaque vie.

On l'a compris : l'argent est un problème politique et la philosophie doit ici être reconduite à sa modeste position de «propédeutique ».

\footnotetext{
${ }^{14}$ Laurence Duchêne, Pierre Zaoui, « Politiques de l'argent », dans Esprit, numéro 1, 2010, p. 189.
} 
